\title{
Decision support for Foodbank South Africa
}

\author{
NM Watson* \\ TJ Stewart ${ }^{\dagger}$ \\ $\mathrm{L} \mathrm{Scott}^{\ddagger}$ \\ Received: 5 November 2012; Revised: 14 February 2013; Accepted: 4 April 2013
}

\begin{abstract}
This paper employs a combined 'soft-hard' OR approach to aid decision making in the area of allocation at a not-for-profit organization, Foodbank South Africa (FBSA), that represents the largest hunger-relief network in South Africa. Two problem-structuring tools, causal mapping and root definitions (RDs) are utilized. Causal mapping is used to identify areas for useful research within FBSA, and gain a greater understanding of the organization in terms of its goals and consequently a good appreciation of the context in which decisions are made. Root definitions are employed to acquire a better understanding of the 'decision-issues' within the allocation system at the Cape Town warehouse. A simulation model is developed to imitate daily allocation decisions, with the end-objective of assisting decision-making by developing a range of allocation policies. A decision support system (DSS) is developed to help FBSA manage their agency database, automate some of the daily allocation decisions and simulate allocation policies.
\end{abstract}

Key words: Cognitive/causal mapping, decision support system, Foodbanking, Operational Research for development, problem-structuring, root definition, simulation.

\section{Introduction}

Hunger and food insecurity are pandemics that are on the rise. Recent statistics from the Food and Agricultural Organization of the United Nations (FAO) [4] indicate that approximately 925 million people 'go hungry', i.e. are undernourished, everyday. More than 239 million of these people live in sub-Saharan Africa, with approximately 14.5 million undernourished people in South Africa [13]. The problem of hunger is perplexing, because the world does produce enough food on a daily basis to provide sufficient nutrition to every person in it [6]. Part of the problem is that a large amount of food is wasted, and foodbanking has developed to help combat hunger by recovering waste food and redistributing it to the hungry as part of its strategy.

\footnotetext{
${ }^{*}$ Corresponding author: Statistical Sciences Department, University of Cape Town, Rondebosch, 7700, South Africa, email: nm.watson@uct.ac.za

$\dagger$ (Fellow of the Operations Research Society of South Africa), Statistical Sciences Department, University of Cape Town, Rondebosch, 7700, South Africa.

${ }^{\ddagger}$ Statistical Sciences Department, University of Cape Town, Rondebosch, 7700, South Africa.
} 
Essentially, 'foodbanking' is the process by which food, that would otherwise be disposed of as waste, is recovered and distributed to hungry people. Although the practice of foodbanking differs somewhat from place to place, the basic building blocks of a foodbanking system are the same everywhere. Usually, a foodbanking system consists of the following four entities.

- A group of donor organizations (retailers, manufacturers, producers), on both a national and local level e.g. Pick 'n Pay.

- A 'foodbank' - usually a warehouse of some kind where food is sorted and stored for delivery.

- A number of authorized social service organizations ('agencies') to which food is donated to feed the hungry.

- People suffering from hunger and food insecurity, who are supported by the agencies.

In general, the social service organizations include: HIV/AIDS homes; soup kitchens; children's homes; school feeding programs and other non-profit organizations. Food is either received or bought (usually at a reduced cost) from the donors and brought to the foodbank where it is sorted and stored. The food received can broadly be classified into perishable goods such as fruit and meat that would become inedible within a few days, and non-perishable goods such as cereals and canned foods that would still last at least a week or more. Perishable goods are typically received and delivered to agencies within 24 hours. Food is either delivered to or collected by agencies on a regular basis - daily or weekly, depending on the agreement between the particular agency and the foodbank.

South Africa's nationwide foodbanking organization, Foodbank South Africa (FBSA), officially opened for business on 2 March 2009 with the commissioning of the Foodbank Cape Town (FBCT) warehouse in Philippi, Cape Town ${ }^{1}$. FBSA is a new organization that is still in the process of establishing its 'identity' (i.e. its short, medium and longterm goals, its organizational structure and its strategies for combating hunger). When the long-term vision of an organization is the elimination of hunger in a country, one can understand that the demand on the organization always exceeds what they can supply. This equates to huge pressure on the allocation policies employed by FBSA and the specific allocation systems at each of their warehouses, both of which are still being developed as FBSA evolves to respond to the many challenges that it faces. Hence there is a great need for decision support in the area of allocation at FBSA.

\section{Problem structuring}

The entry point to working with FBSA came via a series of meetings held with members of its management. Whilst a great deal of information about the organization was gathered during these interactions, there was a lack of clarity as to what sort of intervention would be helpful. When it comes to an Operations Research (OR) intervention in the developing context, there is a heightened need for appropriate action to be taken to solve the problem at hand [11]. However, one must be careful not to act too soon without an adequate

\footnotetext{
1 "FBSA" refers to the organization itself, whilst "FBCT" refers to FBSA's Cape Town warehouse.
} 
understanding of the problem. It was thus decided to utilize problem structuring methods (PSMs) to assist us to identify potentially fruitful areas to focus on, and provide some structure to each potential research problem.

An initial approach was one of first establishing a good relationship with FBSA before even mentioning 'problem-structuring'. Maintaining regular contact with the client is paramount to the success of the intervention [12], and it was thus sought to have regular meetings from the outset. Five areas needing decision support quickly surfaced from discussions with FBSA: developing a comprehensive poverty map of South Africa, determining optimum locations for new foodbanks, vehicle routing, maintenance of their fleet of vehicles and improving their current allocation system. During subsequent meetings, rough cognitive maps of these problems areas were created. From this exercise, it became evident that the first two areas were too large in scope to be tackled as part of this study, whilst FBSA already had someone conducting research into vehicle routing. Of the two remaining areas, improving FBSA's current allocation system held great potential for an OR type intervention and thus it was decided to focus on this problem.

The goal was to use whatever PSM, or parts of PSMs, that were suitable with respect to both their appurtenance to the selected problematical situation and their transparency. Here, 'transparency' refers to how easily the particular methods/tools employed are understood by the client. Mingers and Rosenhead [7, pp. 842] suggest that, in order for PSMs to be effective, they should (1) enable alternative perspectives to be considered with each other, (2) be transparent to a range of participants, (3) operate iteratively, and (4) allow contingent solutions.

These characteristics served as guidelines (a sort of 'check-list') in both selecting and employing the PSMs used in this study. Today, many Operations Researchers combine parts of different PSMs in their problem structuring [3]. Mingers [8] posits that it is both possible and beneficial to abstract pieces of different methodologies at the level of techniques/tools, and use these tools to augment the problem-structuring approach being employed. This is precisely what was done in this study where no particular PSM was applied in its entirety, primarily because it was felt that doing so would be too time consuming and it was evident from very early on in the definition of the problem that a combination of soft and hard OR methods would be needed. Initially, a PSM was sought that would enable us to investigate the organizational structure of FBSA; whilst structuring the allocation system at FBCT called for a PSM that was suited to systems involving humans making decisions. Hence, two specific tools within recognized PSMs were employed as part of a broader problem-structuring process, namely causal mapping (adapted from Strategic Options Decision Analysis (SODA)) [1] and root definitions from SSM [2].

\subsection{Causal mapping of FBSA}

The causal mapping technique employed in this study most closely resembles cognitive mapping, as used in SODA by Eden and Ackermann [1]. Although the cognitive mapping approach was not rigorously adhered to, it did serve as a guideline in creating the causal 
maps in this study ${ }^{2}$. The approach followed was unique in that the maps were initially created solely by the author of this study from information gained from FBSA's website, meetings with FBSA, and documentation obtained from them. It is noted that this is a significant departure from the traditional brainstorming or interview approaches, but it still proved very useful in structuring the problem, a well as strengthening a mutual understanding (between the author and the client) of the organization itself.

A 'top-down' approach was adopted in creating the maps, where the links between concepts were causal in nature. The 'opposite poles' concept from cognitive mapping [1] was used,

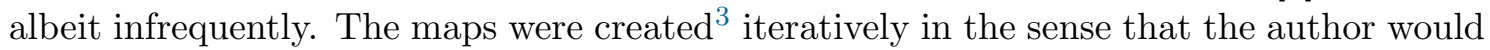
create and present a map to the supervisors of this study who would provide constructive feedback, which would then be incorporated and a subsequent presentation of the improved map made. This process would continue until there was general agreement between the author and the supervisors that the map was ready to be presented to representatives from FBSA. The next step was to hold a meeting with FBSA, in which the maps would serve as a communication tool in an interview-type setting, to stimulate discussion of the issues represented on the map. This was found to be very beneficial to both the author and the representatives from FBSA, one of whom expressed a keen interest in using causal mapping in some of his own work within the organization.

Another significant departure from traditional causal mapping approaches is that the chief outcome of the entire mapping exercise was to ensure that the author had a good understanding of FBSA as an organization in terms of its goals. This was certainly seen to fall under structuring of the problem, as the organization itself serves as the context from which the problem emanated. Thus, understanding the organization's vision, strategic objectives and goals would go a long way towards a good structure of the problem. The 'means-ends' context was used in creating the maps, where different colours for concepts were used to indicate where they belonged on the hierarchy (overall goal, strategic objective, possible action). Concepts were also worded in terms of 'states of being' i.e. they were represented as a desired state of being rather than in the more established 'actionorientation' [1]. For example, instead of a concept reading 'Establish more foodbanks' it would read 'Having a sufficient number of foodbanks'.

Figure 1 represents a small excerpt from a causal map of the 'overall' or 'blanket' goals of FBSA. Note that this map was the final product of the iterative approach described above, thus reflecting the beliefs/worldviews of representatives from FBSA.

The vision of FBSA is to see hunger, food insecurity and poverty eradicated in SA. Following the top-down approach, the next step was to ask the question 'What would be necessary/helpful for FBSA to achieve this state?' In answer to this question, the concepts 'Strong, self-sustaining urban and rural communities' and 'Quality food secured and distributed to the hungry' were developed i.e. FBSA believes that both securing and distributing quality food to the hungry and having strong, self-sustaining urban and rural communities will play an integral part in eradicating hunger, food insecurity and poverty

\footnotetext{
${ }^{2}$ The tutorial 'Getting Started with Cognitive Mapping', provided with Banxia's Decision Explorer Software, proved to be very useful in this regard.

${ }^{3}$ The causal maps in this study were created using the Banxia Decision Explorer (COPE) software, (C)1991-2007 Banxia Software.
} 


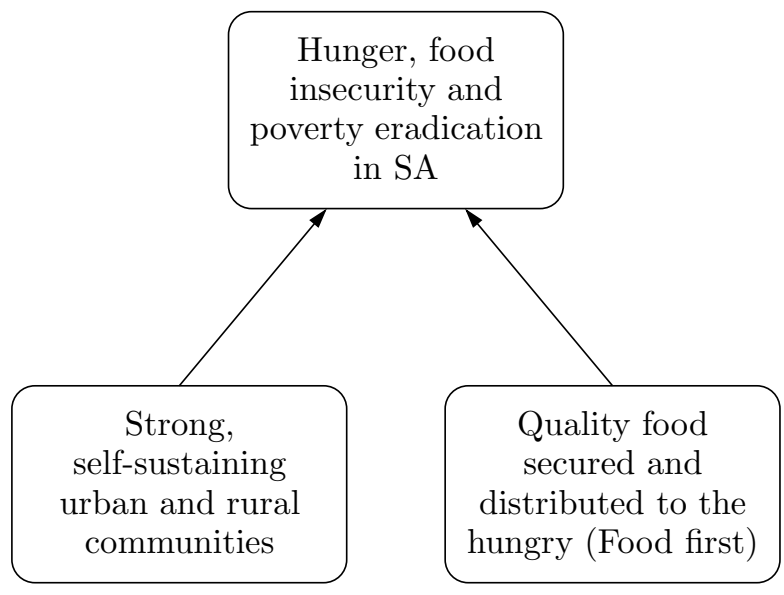

Figure 1: A causal map of the overall goals as outlined by FBSA.

in SA. Both these concepts are long-term goals and thus also form part of FBSA's vision. These two concepts also reflect two pillars of FBSA's strategy — that of the distribution of quality food and community development.

Following the top-down approach a larger causal map of FBSA's overall goals and strategic objectives and a comprehensive causal map of FBSA's overall, strategic and short-term objectives were created ${ }^{4}$. The causal maps yielded a wealth of valuable information about FBSA as an organization and its approach to ending hunger, food insecurity and poverty in $\mathrm{SA}$, and consequently the context in which decisions are made. However, specific attention was placed on FBCT's allocation system in order to structure the problem of improving it. Since SSM places a large emphasis on modelling purposeful activity systems involving human beings making decisions, particularly through the construction of root definitions (RDs) [8], it was decided to utilize RDs to structure the research problem further.

\subsection{Applying SSM's root definitions to FBCT's allocation system}

Within the 'finding out' phase of the SSM process, analysing the intervention itself entails thinking about how the practitioner employs SSM in order to address the perceived content of the problematical situation [8]. Usually, there are three roles that exist in any problematical situation and in this case they are:

- the 'client' - the person or group of people that caused the intervention to happen (FBCT),

- the 'practitioner' - the person/s conducting the intervention (the author of this study), and

- the 'issue owners/stakeholders' - those people who care about or are affected by the current situation and any action taken to improve it (various people involved in FBCT's allocation system).

\footnotetext{
${ }^{4}$ The map of FBSA's overall, strategic and short-term goals is too large to be included here. It is available from the author on request.
} 
Every problematical situation involves people, each acting purposefully with their own worldview [8]. A RD is essentially a written statement that describes a problematical situation as a purposeful activity system from a particular person's viewpoint or worldview. What is crucial to understand here is that there may be copious RDs for any problematical situation $[8]^{5}$, since each RD is contingent upon the worldview of one person (or group of people) who have an interest in the system. RDs are invariably created by considering the elements of the mnemonic 'CATWOE', illustrated in Table 1 (adapted from [2]) which helps to define the purposeful activity system under investigation. The 'PQR' formula [8]: Do ' $\mathrm{P}$ ' by ' $\mathrm{Q}$ ' in order to help achieve ' $\mathrm{R}$ ' can also be used to define the system by providing answers to the questions: Do 'What'? (P), 'How' (Q) and 'Why' (R).

\begin{tabular}{cll}
\hline C & Customer $/ s$ & Who would be the victims/beneficiaries of the activity? \\
A & Actor $/ s$ & Who would do the activities? \\
T & Transformation Process & What is the purposeful activity? \\
W & Worldview & What view of the world makes this definition meaningful? \\
$\mathbf{O}$ & Owner $/ s$ & Who could stop this activity? \\
$\mathbf{E}$ & Environmental Constraints & What constraints in the environment are taken as given? \\
\hline
\end{tabular}

Table 1: A summary of the CATWOE mnemonic.

It was decided to use RDs to gain a better understanding of the allocation system at FBCT. First, the relevant stakeholders in the allocation system were identified. Then their roles within the system were defined, and a RD for each stakeholder was formulated. A comparison between the RDs of each stakeholder was conducted, in the hope that this would shed more light on the problem. Subsequent to this, criteria were developed against which the performance of the allocation system should be measured.

The allocation system at FBCT consists of the following stakeholders ${ }^{6}$, and their roles within the system:

- Food Donors/suppliers: the organizations/individuals who donate food to FBCT.

- FBCT Ground-staff: the people working at FBCT on a daily basis.

- FBCT Warehouse manager: oversees the operation of the FBCT warehouse.

- FBSA National Agency Coordinator (NAC): has to meet the agencies supported by FBCT each quarter and explain the current allocation policy to them.

- Social Welfare Organizations (agencies): the organizations in and around CT that are supported by FBCT.

After the initial meetings held with representatives from FBSA, subsequent meetings with representatives from FBCT were convened and the author spent three full working days at the FBCT warehouse where the allocation system was observed. Subsequent to this, RDs for each of the stakeholders were constructed and then compared. The RDs across all stakeholders were similar. All recognized that those who stand to benefit from the system

\footnotetext{
${ }^{5}$ Hence the metaphor 'root' that alludes to the fact that each RD is but one core way of describing the system.

${ }^{6}$ Note that this list is not exhaustive - it is intended to represent a cross-section of the stakeholders in FBCT's allocation system that fulfil different roles, in order for us to appreciate the influence of their different worldviews in gaining a better understanding of the problem. Also, to formulate a RD for every possible stakeholder would be too time consuming and unnecessary for the purposes of this study.
} 
are the agencies (customers), and the system could be changed by the management at FBCT (owners), whilst the management and ground staff at the FBCT warehouse are the people who actually 'do' the allocation system (actors). The differences existed in people's worldviews (which is to be expected), the transformation process and the environmental constraints.

The stakeholders who represent FBCT (ground-staff, general manager and the FBSA NAC) are all concerned, to different degrees, about the effectiveness and efficiency of the allocation system as a whole. The food donors/suppliers are chiefly interested in the efficiency with which their waste food is taken from them, whilst the FBSA NAC and the agencies are primarily concerned with the fairness and accuracy of the allocation system. Thus, there may potentially be conflicting interests. The issues of fairness and accuracy add significant complexity to the problem of improving the allocation system in that incorporating them may result in more time-consuming allocations and thus a reduced efficiency of the system - which is what the FBCT general manager (and management in general) would not want. However, seeking to improve just the efficiency of the system may result in biased and inaccurate allocations - which is what the FBSA NAC and the agencies would not want. This tension between the two issues is something that any solution will have to address.

The transformation process or change for all the stakeholders, barring the food donors or suppliers, is essentially the improvement of the current allocation system into one that is more accurate, efficient and fair. All of the stakeholders with this transformation have interests in the accuracy, efficiency and fairness of the allocation system, albeit with different primary concerns.

The environmental constraints for all stakeholders, except the agencies (who simply have to accept the allocation system as it stands), essentially involve the FBCT warehouse's resources - whether it be the number of staff, equipment or warehouse or transport capacity. These are constraints that are assumed to be relatively inflexible for the time being as FBSA is a not-for-profit organization that does not have a limitless budget to hire more staff or purchase more equipment or trucks, and there are no immediate plans to relocate to another larger warehouse. Another crucial constraint is the amount of donated food that FBCT receives on a daily basis — which is invariably far too little to satisfy the needs of the agencies presently being supported. This is also a relatively inflexible constraint in that, although the quantity of donated food is gradually increasing over time (as FBSA sources more donors/suppliers), the list of social welfare organizations requesting support is also growing. At present there are more than 2000 organizations across South Africa that are on a waiting list to receive support from FBSA. These constraints will have to be incorporated into any proposed solution.

It is important to outline the criteria by which the performance of the allocation system will be judged, as these will serve as good measures of the appropriateness of the solution. The 'three E's' (adapted from [8]) that are pertinent for any system are:

- Efficacy - criteria for efficacy indicate whether the transformation is working

- Efficiency - whether the transformation is being achieved using a minimum of resources 
- Effectiveness — whether the transformation is helping to achieve some longer-term or higher-level goal

The above framework was used as a guide in developing relevant performance criteria for the allocation system at FBCT. The higher-level goal for this transformation would be to improve the support provided by FBCT to the agencies. The primary intended outcomes of the transformation are improving the accuracy, efficiency and fairness of the allocation system. This would equate to a balanced percentage of satisfied needs across all agencies, a reduction in the time taken to perform allocations, and a higher percentage of satisfied needs for particular food types across all agencies. Any proposed changes to the allocation system should not exceed the current resource use, and changes that reduce the amount of resources used whilst either maintaining the performance of the current allocation system or improving it would be desirable. These criteria provide us with useful measures of the appropriateness of any proposed solution to the problem of improving FBCT's current allocation system. Any solution will also be judged according to whether it fits into the strategic and overall goals of FBSA (effectiveness), which are important to consider.

Applying the RDs helped us obtain a better perspective of where FBCT's allocation system fits into the 'bigger picture' of FBSA and its vision and goals - FBSA wants to see effective and efficient allocation of food to the agencies it supports, but would like to do this within their current resource constraints and without compromising the efficiency of the current allocation system.

\section{Developing decision support}

Rosenhead posits that a successful application of a PSM in an OR intervention generates collaborative action [among stakeholders] towards a desired future [10, pp. 764]. Whilst the application of the soft OR tools was useful in structuring the problem of improving FBCT's allocation system, they were not suitable to help develop a solution to the problem. Hence, they were not used to facilitate negotiation among the various stakeholders towards an agreed solution. In order to improve the allocation system at FBCT, it needed to be modelled and simulated. Building a simulation model of the allocation system would help us understand the allocation decisions made on a daily basis, and also allow us to investigate the effect of any proposed changes (i.e. new or different allocation decisions) to the system.

\subsection{Simulation model}

In developing a model of FBCT's allocation system, two primary areas requiring decision support were identified: the daily allocation decisions facing the floor managers at the FBCT warehouse and the allocation policies employed by FBCT. It quickly became apparent that these two areas are related, as the allocation policy employed directs the daily allocation decisions which affect the performance of the system which then determines whether a change to the current allocation policy is needed. This relationship is depicted in Figure 2 which shows a broad outline of the simulation model that serves as the basis for the simulation study. 


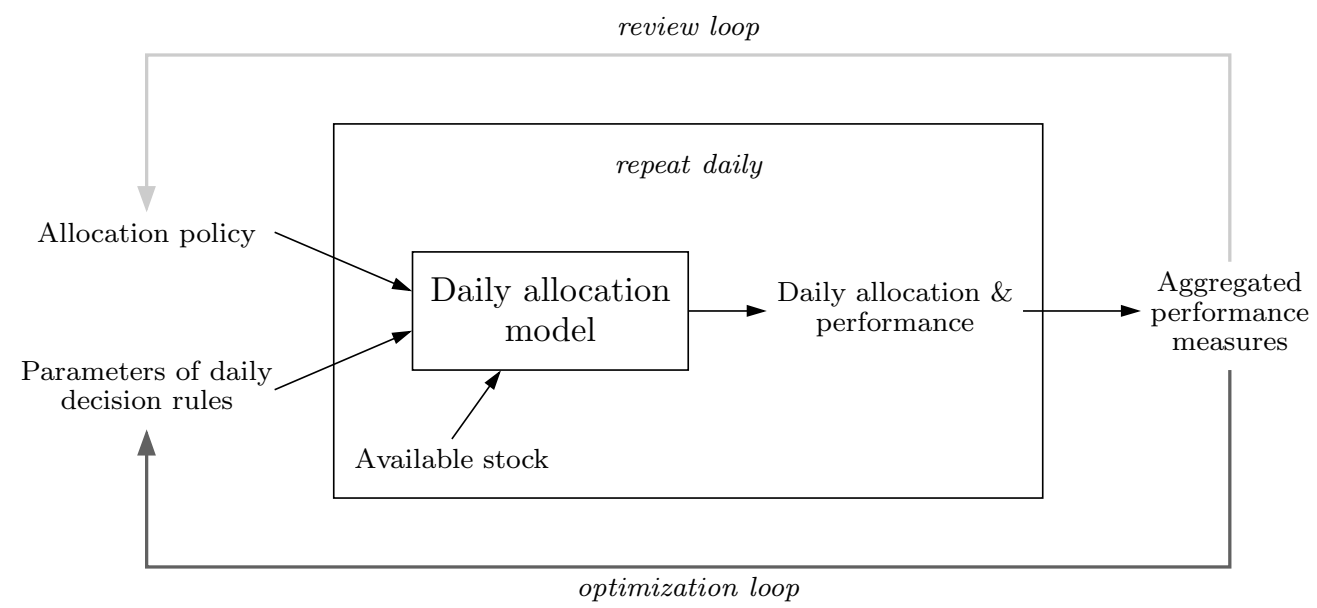

Figure 2: A broad outline of the simulation model.

The model consists of two 'layers' or sub-models - one within the other. The first, inner layer is the allocation system at FBCT. It will have as its inputs the available stocks of perishable and non-perishable goods and will simulate the daily allocation decisions that are made. These decisions are expressed as linear algebraic equations in the model and are governed by decision rules (DRs). The two major daily allocation decisions are:

1. What types of food go to which category of agency. The DR here is based on the needs and cooking facilities of each type of agency and is predetermined. For example, an educare that has no cooking facilities has no use for raw meat. Likewise, allocating large amounts of vegetables to agencies that have no cooling facilities would result in a waste of food ${ }^{7}$.

2. How much of each type of food is given to each agency. This DR is the intended outcome of the allocation. For example, equal allocation across all agencies versus equal percentage of need satisfied across all agencies. It will be one of the major focus areas of the simulation model.

These DRs are functions of variable parameters, and any subset of them collectively forms an allocation policy. Thus, the DRs with their parameters also form part of the input into the daily allocation model, as reflected in Figure 2. The first layer of the simulation model is repeated daily, each repetition representing a separate simulation. The second, outer layer, consists of two parts, namely optimization and review.

Optimization. The aim of simulating the daily allocation system is to find the values of the parameters incorporated within the DRs being modelled that correspond to the best performance of the system. This is reflected in Figure 2 by the optimization loop where loop indicates that many simulations will be run in order to determine good parameter values. Once a specified number of simulations of the inner layer have been run, aggregated performance measures of the system are calculated. These aggregated performance measures

\footnotetext{
${ }^{7}$ Whilst it would be worthwhile investigating the affects of changes to the classification of which food types are allocated to which agencies, for the purposes of this study it was decided to leave the classification as it stands.
} 
are used to determine a set of good parameter values of the DRs for each allocation policy considered.

Review. The outer layer has as its focus longer-term allocation decisions, in particular the decision about which DRs to use to form an allocation policy. This is indicated by the review loop in Figure 2. The optimization loop outputs an optimal allocation policy for a specific set of DRs. However, FBCT may well change the set of DRs that make up an allocation policy with a view to improving the allocation system. Such an event is catered for in the review loop. The aim for this part of the simulation would be to derive a range of allocation policies, with their corresponding performance measures, by investigating changes to the set of DRs that constitute an allocation policy. This range of policies could be considered by FBCT when reviewing their operating allocation policy.

In reflecting on Pidd's guidelines for the type of simulation study to be conducted [9], the incoming donations will be modelled stochastically, whilst time will be moved forward in fixed increments of one day. Each day will be an event, with the amounts of donated and allocated foods modelled as discrete random variables. Other elements of the model (such as the classification of what types of foods are allocated to which categories of agency) are predetermined and thus deterministic. Hence, a stochastic discrete-event simulation model in which time is moved forward in fixed increments was developed. The simulation model serves to develop decision support in three primary ways. It will be utilized to perform daily allocations at the FBCT warehouse to optimize the accuracy, efficiency and effectiveness of the allocation system; provide members of FBSA with a tool to review allocation policies by simulating them and investigating their performances, as two components of the DSS; and conduct a simulation study of a number of allocation policies with a view to developing a range of 'optimal' allocation policies to be considered by FBSA for future use.

The simulation model presented here is a representation of the allocation system at FBCT. With this in mind, a representative list of agencies was constructed from information derived from FBCT's agency database by determining the average number of agencies allocated food each day (42), each category of agency allocated food each day, and people supported per agency of each category of agency allocated food each day.

Due to limitations to both the scope of and time available for this study, only two allocation models (policies) were investigated in a simulation study ${ }^{8}$, namely a max-min allocation policy, and a number-based allocation policy.

Let

$Y_{i} \quad$ be the amount of food type $i$ available (stock) to be allocated,

$X_{i p}$ be the amount of food type $i$ allocated to agency $p$,

$T_{p} \quad$ be the the number of people supported by agency $p$,

$W_{i}$ be the total number of people allocated food type $i$ across all agencies,

$S_{i j} \quad$ be a binary variable that indicates whether food type $i$ can be allocated to agency category $j$,

\footnotetext{
${ }^{8}$ There is certainly room for further simulation studies to be carried out within the framework that Figure 2 provides.
} 
$v_{i}$ be the nutritional value of the amount of allocated food $i$, and

$V_{p}$ be the total food-value per person in agency $p$,

where $i=1, \ldots, 12, j=1, \ldots, 9$ and $p=1, \ldots, s$. It is necessary to ensure that $X_{i p} \geq 0$ for all values of $i$ and $p$. Similarly, $\sum_{p=1}^{s} X_{i p} \leq Y_{i}$ for $i=1, \ldots 12$. The total value per person in agency $p$ is calculated as $V_{p}=\sum_{i=1}^{12} v_{i} X_{i p} / T_{p}$.

For the max-min allocation policy formulation the following need to be considered. Let

$l_{i p} \quad$ be the lower bound placed on quantity of food type $i$ allocated to agency $p$, and

$u_{i p}$ be the upper bound placed on quantity of food type $i$ allocated to agency $p$.

The pro-rata quantity of food type $i$ to be allocated to agency $p$ is given as $R=\frac{X_{i p} \times T_{p}}{W_{i}}$. Then the bounds placed on the quantity of food type $i$ allocated to agency $p$ are ${ }^{9} \frac{R}{l_{i p}} \leq$ $X_{i p} \leq u_{i p}(R)$. Let $D$ be the adjustable cell representing the maximum minimum foodvalue per person of the allocated quantities of food across all agencies to be maximized, and let $D \leq V_{p}$ for $p=1, \ldots s$. Then objective is

Maximise $D$

$$
\begin{aligned}
& \text { subject to } D \leq V_{p}, \quad p=1, \ldots s, \\
& X_{i p} \geq \frac{R}{l_{i p}} \quad p=1, \ldots, s, \quad i=1, \ldots 12, \\
& X_{i p} \leq u_{i p}(R) \quad p=1, \ldots, s, \quad i=1, \ldots 12, \\
& \sum_{p=1}^{s} X_{i p} \leq Y_{i}, \quad i=1, \ldots 12 \text {, } \\
& X_{i p} \geq 0 \quad p=1, \ldots, s, \quad i=1, \ldots 12 .
\end{aligned}
$$

For the number-based allocation policy formulation the following need to be considered. Let $\mathrm{A}_{i p}$ be agency $p$ 's allocation binary variable for food type $i$ and $\mathrm{F}_{i p}$ be agency $p$ 's allocation factor for food type $i$. Furthermore, $\mathrm{F}_{i p}=\frac{\mathrm{A}_{i p} \times T_{p}}{W_{i}}$ and $X_{i p}=\mathrm{F}_{i p} \times Y_{i}$ for $i=1, \ldots 12, p=1, \ldots s$.

The max-min allocation policy attempts to balance the $\mathrm{kcal} /$ person value across all agencies, i.e. maximize the minimum $\mathrm{kcal} /$ person value across all the agencies. Performing such an allocation makes sense as the nutritional quality of the allocated food is important. This nutritional quality of food is what corresponds to FBSA's desire to improve the accuracy of their allocations. The number of people being supported by each agency is included by constraining the amount of each type of food allocated to an agency to be greater than or equal to half of its pro-rata amount, but less than or equal to double this amount.

The number-based allocation policy is essentially a representation of the allocation system currently in use at FBCT. Available stock is divided amongst agencies exclusively according to the number of people being supported by each agency. This model is not of an optimization-type. The way in which food is allocated is based upon pre-determined decisions (like the classification of what food types are to be allocated to which agency types), which for the purposes of this simulation study were not being varied.

\footnotetext{
${ }^{9}$ Note that the default value for both the lower and upper bounds is ' 2 '.
} 
For each policy, the decisions regarding the quantity of each food type to be allocated to each agency are included in the model by defining allocation factors for each food type for each agency. Each allocation factor is calculated by taking the product of the allocation binary variable corresponding to the agency's category and the number of people supported by that agency. This value is then divided by the total number of people across all the agencies who are to be allocated that food type to get the corresponding allocation factor. The obvious difference between the two models lies in their objective functions and associated constraints. Since the demand for food currently far exceeds what FBCT can supply, all their stock is allocated i.e. no perishable food is left unallocated on a daily basis.

The performance of the allocation system (i.e. each allocation policy) will be measured as a function of five performance measures, namely:

1. the average $\mathrm{kcal} /$ person value in each agency,

2. the average minimum $\mathrm{kcal} /$ person value in each agency,

3. the average percentage of agency's target nutritional need satisfied,

4. the average variance in percentage of agency's target nutritional need satisfied, and

5. the average number of agencies receiving less than $50 \%$ of their target percentage need.

These statistics essentially measure the accuracy and fairness of the allocation system, which are two of the primary areas needing improvement that were identified in the problem structuring exercise.

The average kcal/person value in each agency is a good measure of the overall performance of each allocation policy. At present, FBSA's allocation target is 'one meal per person per day' for each agency, which equates to approximately 700 kcal per person. Thus, the closer this value is to the target value of $700 \mathrm{kcal}$, the better the performance of the policy. The average minimum kcal/person value in each agency focuses on the minimum $\mathrm{kcal} /$ person value across all agencies. Ideally, FBSA would want this value to be much the same as the average $\mathrm{kcal} /$ person value, as this would indicate a low variance in the $\mathrm{kcal} /$ person values across all the people being supported by the agencies. One would expect the max-min policy to outperform the number-based policy here, as maximizing the minimum kcal/person value is its objective. The average percentage of agency's target nutritional need satisfied presents a more salient view of the performance of each policy. Whilst it is useful to know how each policy performs with respect to the individuals being supported by each agency, the interpretation of the previous two statistics can be slightly ambiguous in the sense that they measure the performance of each policy across all agencies, and do not focus on performance with respect to each agency. The average percentage of an agency's target nutritional need satisfied measures the performance of each policy with respect to each agency being supported, and thus provides us with a more holistic performance measure. Obviously, FBSA would want this measure to be as close to $100 \%$ as possible. The average variance in percentage of agency's target nutritional need satisfied measures the variation in the percentage of an agency's need that was satisfied by each allocation policy and is an important indication of the overall performance of each policy. Ideally, FBSA would want this statistic to be as close to ' 0 ' as possible. The average number of agencies receiving less than $50 \%$ of their target percentage need gives us 
another indication of how each policy is performing. It is obvious that FBSA would always want this statistic to be zero, thus ensuring that, at the very least, they are satisfying half of each agency's needs.

The performance measures focus on the nutritional value of the food received by agencies. This is seen as a more precise measure of the value of an allocation than just kilograms of allocated food. Variances in kilograms may result in significant differences in the nutritional value, and consequently the satisfaction of hunger, at a particular agency. For example, although agency $\mathrm{A}$ and $\mathrm{B}$ may receive the same percentage of needed kilograms of food, agency A may receive only dry goods, whilst agency B may receive a combination of fruit, vegetables, meats and dry goods that is of far greater nutritional value than agency A's allocation.

In order to incorporate the nutritional value performance measure into the simulation model, an average nutritional value $(\mathrm{kcal} / \mathrm{kg})$ for each food category was calculated by computing the average $\mathrm{kcal} / \mathrm{kg}$ value across the most common foods in each category. The target nutritional value for each agency depends on the average daily human nutritional requirements, the target level of need to be satisfied, and the number of people being supported by the agency. According to the FAO [5], the average human being needs approximately 2000-2200 kcal per day to meet basic nutrition needs (for simplicity, a figure of $2100 \mathrm{kcal}$ is used). Thus, if FBCT aims to provide one meal per day per person being supported by an agency (i.e. 33.3\% of need satisfied or 700 kcal per person) that supports 50 people, then the total nutritional target for the agency would be $35000 \mathrm{kcal}$ per day.

The simulation experiments focused on simulating the max-min and number-based allocation models for different lengths of time, observing and then comparing their individual performances. It was decided to consider three simulation experiments of different time periods: short-term (one month), medium-term (one year) and long-term (five years). It is important to consider different simulation periods as the performance of each of the models may vary over time. Whilst it is acknowledged that shorter simulation runs inevitably lead to more variable results, the rationale behind including different lengths of simulations was that there was value in observing the extent of the difference in variance of the results obtained for each of the simulation experiments. Another possible simulation strategy may be to settle on one simulation length (at least a year) and run several experiments with that length and then compare the results. The results from each of the three simulation experiments are summarized in Table 2.

A number of conclusions can be drawn from examining the results of the three simulation experiments. One policy is not categorically better than the other. Each policy has its advantages and disadvantages:

- The max-min policy consistently outperformed the number-based policy with respect to the average $\mathrm{kcal} /$ person and average minimum $\mathrm{kcal} /$ person values.

- The number-based policy consistently outperformed the max-min policy with respect to the average percentage of agency's target nutritional need satisfied and average number of agencies receiving less than 50\% of target percentage need values.

- Both policies exhibited too high a value for the average variance in percentage of 


\begin{tabular}{lccc}
\hline Allocation policy & \multicolumn{3}{c}{ Max-min } \\
Simulation experiment (length) & Short & Medium & Long \\
\hline Avg. kcal/p & 574.85 & 660.14 & 666.97 \\
Avg. min. kcal/p & 344.93 & 354.54 & 359.39 \\
Avg. percentage of agency's target nutritional need satisfied & 62.26 & 68.82 & 69.71 \\
Avg. variance in percentage of agency's target nutritional need satisfied & 20.66 & 32.43 & 32.80 \\
Avg. num. of agencies receiving less than 50\% of target percentage need & 22 & 18 & 18 \\
\hline Allocation policy & \multicolumn{3}{c}{ Number-based } \\
Simulation experiment (length) & Short & Medium & Long \\
\hline Avg. kcal/p & 534.57 & 632.32 & 640.44 \\
Avg. min. kcal/p & 191.35 & 201.07 & 201.22 \\
Avg. percentage of agency's target nutritional need satisfied & 74.11 & 84.31 & 85.33 \\
Avg. variance in percentage of agency's target nutritional need satisfied & 18.78 & 34.85 & 34.21 \\
Avg. num. of agencies receiving less than 50\% of target percentage need & 15 & 14 & 13 \\
\hline
\end{tabular}

Table 2: Results from the three simulation experiments.

agency's target nutritional need satisfied, with the number-based policy initially performing better than its counterpart. In the long run, the max-min policy demonstrated a slightly lower value than the number-based policy for this performance measure.

The decision on which allocation policy to implement in the future is entirely up to FBSA. Both policies can be utilized by FBSA, the choice of which one will depend on which of the three criteria of accuracy, efficiency and fairness FBSA deem to be most important. Should they wish to maintain the efficiency of the allocation system, the number-based policy would be the best option. However, should they wish to improve the accuracy and fairness of the allocation system then the max-min policy should be adopted. Since the accuracy and fairness of the allocation system are the two factors that have the greatest influence on the agencies supported by FBCT, it is recommended that the max-min policy be adopted.

\subsection{Decision support system - FAST}

The DSS FAST (FBSA's Agency Allocation Support Tool) was created to assist FBSA with allocation decisions. It comprises two major functions: a database management system and daily allocation tool, and a simulation model to investigate the long-term performances of different allocation policies. These two functions are clearly related to the inner and outer layers of the broader simulation model presented in Figure 2.

FAST as a database management system and daily allocation tool was developed to help aid the decision-making of the managers involved in performing daily allocations at FBSA (i.e. at any one of the FBSA warehouses countrywide), with the hope of improving both the accuracy and efficiency of the allocation system as a whole. The 'FAST Homepage' userform is displayed in Figure 3(a). It presents the user with the two major areas of the allocation system, namely Agencies and Allocation. The Agencies frame presents the user with the options of adding a new agency, changing an existing agency's details or removing an existing agency. Upon selection, FAST guides the user through each option 
via a series of userforms.

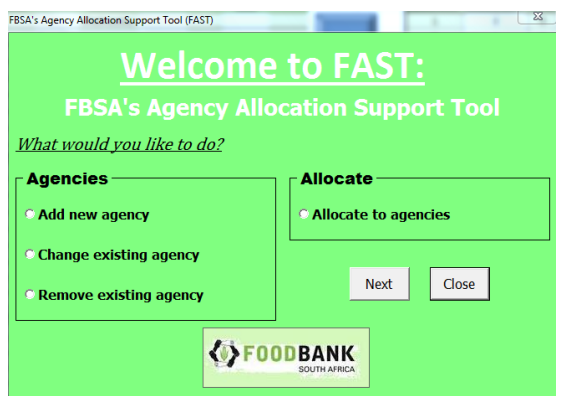

(a) A screenshot of the home page of FAST.

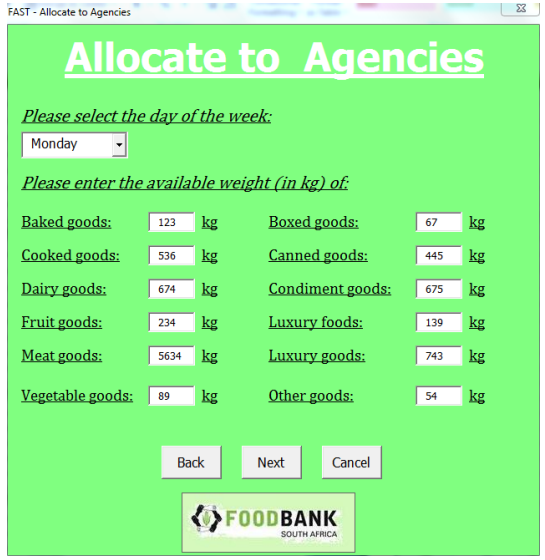

(b) A screenshot of the allocation userform of FAST.

Figure 3: Userforms of FBSA's Agency Allocation Support Tool (FAST).

Upon selecting the 'Allocate to agencies' option in the 'Allocate' frame and pressing the 'Next' button, the 'FAST - Allocate to Agencies' userform appears (see Figure 3(b)). The user is prompted to select the day for which they would like to perform an allocation and is required to enter non-negative integer values for the amounts of each type of food they want to allocate. These values will usually correspond to the stock that is available at the foodbank at the time of allocation. Should the user wish to allocate only perishable or nonperishable items, they can leave the right or left column values at 0 respectively. Once the user has filled in the allocation values, they can perform either of the two allocation types. After the model performs the selected allocation, FAST outputs the resulting allocation food values for each agency on the spreadsheet corresponding to the day selected, as in Figure 4. Thus, the user can identify how much of each type of food (rounded off to the nearest kilogram) to give to each agency on that day.

Agency
\begin{tabular}{|l|c|c|c|c|c|c|c|c|c|c|c|c|}
\hline Bthlone Youth Development food Cooked food Dairy & 21 & 32 & 32 & 23 & 29 & 24 & 27 & 28 & 27 & 26 & 38 & 21 \\
\hline Care Haven & 28 & 43 & 43 & 31 & 38 & 33 & 36 & 37 & 36 & 34 & 51 \\
\hline Siyaphambili Orphanage & 126 & 194 & 193 & 138 & 172 & 147 & 163 & 167 & 163 & 155 & 28 \\
\hline Urban \& Rural Development & 2367 & 3640 & 3620 & 2584 & 3224 & 2760 & 3060 & 3130 & 3060 & 2909 & 426 \\
\hline Life Community Welfare & 168 & 258 & 257 & 184 & 229 & 196 & 217 & 222 & 217 & 207 & 320 & 2367 \\
\hline
\end{tabular}

Figure 4: An example of the allocation values in the Excel spreadsheet of FAST.

FAST was developed as a simulation model to investigate the long-term performances of different allocation policies to assist FBSA in medium to long-term decisions regarding which allocation policy they should employ. FAST is intended to perform daily allocations, i.e. to assist the user in making daily allocation decisions. The decision support provided by FAST as a DSS for reviewing allocation policies is intended for those decisions pertaining to the adoption/removal/changing of allocation policies at FBSA, i.e. decisions that will occur on a far less frequent basis - perhaps only a few times per year. Thus this function of FAST is one that the user would spend a considerable amount of time utilizing as they simulate and examine results, then re-simulate and re-examine results, perhaps 
make some adjustments to an allocation model, and then re-simulate etc. The purpose of this function of FAST is to provide the user with a simple tool to simulate a particular allocation policy for whatever length of time they desire ${ }^{10}$, and present them with the same descriptive performance measures of that policy as utilized in the simulation study.

Figure 5 depicts the Simulation Homepage. It displays the number of days to simulate, the number of simulations to run, the nutritional value (kcal $/ \mathrm{kg}$ ) of the 12 food types allocated by FBSA and simulation results from the most recent simulation run displaying the performance measures for both allocation policies.

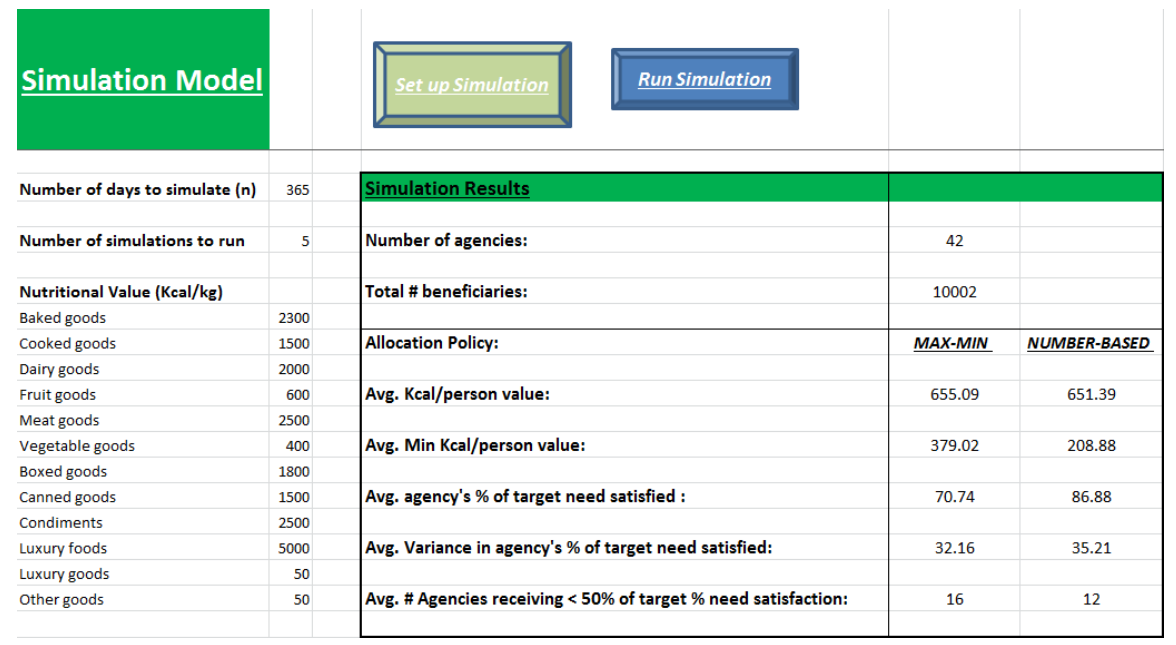

Figure 5: A screenshot of the simulation homepage.

To set up a simulation, the user can simply select the Simulation Setup button which will display a userform where the user can choose both the number of days (1 to 365) and number of iterations for which they wish to simulate the model. To run a simulation, the user can select the blue Run Simulation button. After selecting the button, some time (from minutes to hours, depending on the length of the period to be simulated and the number of iterations) must be allowed for the simulation to run. Once the simulation is complete, the Simulation Results table will be updated with the new information for the user to examine.

\section{Conclusions and recommendations}

This study applied a combined soft-hard OR approach in a developing context in the area of the allocation at a not-for-profit organization, FBSA. Causal mapping helped both the author of this study and members of FBSA to gain a greater understanding of FBSA in terms of its goals, and consequently a good appreciation of the context in which decisions in the organization are made. The construction of RDs helped the author to better comprehend the decision-issues within the allocation system at $\mathrm{FBCT}$, by exploring the different worldviews of various stakeholders of the system. The insight gained from

\footnotetext{
${ }^{10} \mathrm{~A}$ Monte Carlo simulation procedure is employed, where the user can simulate a certain number of days (a maximum of 365) for as many iterations as they desire.
} 
structuring the problem was critical in recognizing the need for a simulation study of the allocation system at FBCT, guiding the development of the simulation model, and interpreting the results of the consequent simulation experiments.

The simulation model developed was a good representation of the allocation system at FBCT, and proved useful in investigating the performances of allocation policies to address the accuracy, efficiency and fairness of FBCT's allocation system. FAST as a DSS for performing daily allocations provides FBSA with a tool with which they can manage their database of agencies and perform daily allocations (automating many of the allocation decisions involved in this process), decreasing the time taken to perform these allocations and hence improving the efficiency of the system. FAST as a DSS for reviewing allocation policies can assist decision makers at FBSA/FBCT to review the performances of current and future allocation policies. FAST is transparent and easy to use, and the management at FBCT trusts it enough to test it and intend to implement it on a national level. Overall, the assistance provided by FAST can help them improve the accuracy, efficiency and fairness of the allocation system as a whole.

The simulation study helped to investigate the robustness of the max-min foodvalue and number-based equal allocation policies. The results showed that both policies have their advantages and disadvantages when compared to one another, with neither policy being conclusively better than the other. In order to improve FBCT's service to their beneficiary agencies, the max-min policy is recommended. Whilst only these two policies were considered in this study, they can both be utilized by FBSA within their current resource constraints. The results obtained from the simulation study can assist the decision makers at FBSA/FBCT to decide on which policy to adopt in the future.

There is large scope for further research to be conducted in the area focused on in this study, and a few pertinent areas are included here.

- Investigate the effects of changing the food classifications to agencies on the performance of the two policies considered in this study.

- Incorporate a measure of the nutritional quality (in terms of providing a variety) of food allocated to agencies.

- Incorporate the financial donations received by FBSA as an input into the model, with a view to exploring the purchase of extra food in order to increase the overall satisfaction of agencies' needs.

- Incorporate the time taken to perform allocations into the model as a performance measure.

The managers at FBCT found FAST to be useful and transparent. They plan on employing it nationally in each of FBSA's five warehouses after familiarising themselves with it. The most recent feedback indicates that FBSA has not done so as they have not been able to allocate a member of staff to drive this process. Thus, further research would need to start with assisting FBSA to roll-out FAST on a national level and then evaluate how useful it is. However, it is evident that FBSA views the work conducted in this study as being helpful to them on a practical level. On a theoretical level, it should serve as a useful contribution to ORD research in similar areas of need in South Africa and the world. 


\section{References}

[1] Ackermann F \& Eden C, 2001, SODA: Journey making and mapping in practice, Chapter 3 in Rosenhead J And Mingers J (EDS.), Rational analysis for a problematic world revisited, Wiley, Chichester.

[2] Checkland P, 2001, Soft Systems Methodology, Chapter 4 in Rosenhead J and Mingers J (Eds.), Rational analysis for a problematic world revisited, Wiley, Chichester.

[3] Eden C \& Ackermann F, 2006, Where next for problem structuring methods, Journal of the Operational Research Society, 57(7), pp. 766-768.

[4] Food and Agricultural Organization of the United Nations, 2010, Global hunger declining, but still unacceptably high, [Online], [Cited: $23^{\text {rd }}$ July 2012], Available from http://www.fao.org/ 62DE99A3-6860-4CFB-ADF5-F2983303379012/al390e/al390e00.pdf.

[5] Food and Agriculture Organization of the United Nations, 2010, Energy requirements and expenditure, [Online], [Cited: $9^{\text {th }}$ September 2010], Available from http://www.fao.org/docrep/ U8480E/U8480E04.html.

[6] Food and Agricultural Organization of the United Nations, 2009, The state of food insecurity in the world 2009, [Online], [Cited: 10 ${ }^{\text {th }}$ May 2010], Available from www.fao.org/docrep/012/ i0876e/i0876e00.htm.

[7] Mingers J \& Rosenhead J, 2006, Problem structuring methods in action, in: White L, Evaluating Problem-structuring methods: Developing an approach to show the value and effectiveness of PSMs, Journal of the Operational Research Society, 57(7), pp. 842-855.

[8] Mingers J \& Rosenhead J, 2001, Rational analysis for a problematic world revisited, $2^{\text {nd }}$ Edition, Wiley, Chichester.

[9] PIDD M, 1987, Simulating automated food plants, Journal of the Operational Research Society, 38(8), pp. 683-692.

[10] Rosenhead J, 2006, Past, present and future of problem structuring methods, Journal of the Operational Research Society, 57(7), pp. 759-765.

[11] Stewart TJ, 2005, Operational research for development in Africa, Technical report, Department of Statistical Sciences, University of Cape Town, Cape Town.

[12] TAYlor AJ, 1982, Some experiences in planning a distribution system, Journal of the Operational Research Society, 33(10), pp. 891-898.

[13] World Hunger Education Service, 2010, World hunger facts 2010, [Online], [Cited: 5 May 2010], Available from www.worldhunger.org/articles/Learn/world. 